\title{
THE ABSORPTION OF SHORT-CHAIN FATS BY PREMATURE INFANTS
}

\author{
BY

\begin{abstract}
SELMA E. SNYDERMAN, SOLEDAD MORALES and L. EMMETT HOLT, Jr. From the Department of Pediatrics, New York University College of Medicine, and the Children's Medical Service, Bellevue Hospital, New York
\end{abstract}

(RECEIVED FOR PUBLICATION AUGUST 7, 1954)

That premature infants commonly exhibit defective fat absorption has been known for many years (Rubner and Langstein, 1915; Muhl, 1923; Tidwell, Holt, Farrow and Neale, 1935). This steatorrhoea is, however, masked and the excess of unabsorbed fat does not separate out in the gross or in microscopically detectable globules.

Efforts to compensate for the caloric deficit caused by this unabsorbed fat have followed several lines, such as low-fat feedings (Gordon and McNamara, 1941), homogenization (Morales, Chung, Lewis, Messina and Holt, 1950b) and emulsifying agents (Snyderman, Morales, Chung, Lewis, Messina and Holt, 1953) to facilitate fat absorption and the use of the more readily assimilable unsaturated fats (Tidwell et al., 1935). The present authors have also shown that increasing the fat intake improved absolute absorption (Morales et al., 1950a) and did not produce deleterious effects.

A further approach to the problem was suggested by the work of Frazer (1946) which indicated that short-chain fats were absorbed through the portal circulation rather than by the lacteals. It seemed possible that the absorption defect in the premature infant might be confined to the lacteal mechanism and that portal absorption would be quite normal. In this event the defect in fat absorption could be overcome simply by feeding short-chain fats.

Observations of this kind were therefore made, using tributyrin and triacetin to replace butter fat in feeds.

\section{Experimental}

Subjects. Six premature infants were used in the present study. They varied in weight from 1,350 to $2,010 \mathrm{~g}$. and in age from 5 to 43 days at the beginning of the study.

Feeds. A stock formula consisting of skim milk, dextrimaltose and water was given to all these infants. The fat was weighed just before the feed and fed by gavage. This was washed down by part of the formula. The remainder of the formula was then given by nipple. This method was adopted in order to be certain that each infant received his entire quota of fat. The formula had the following caloric composition: protein $18^{\circ}$, fat $34 \%$ and carbohydrates $48 \%$, and was given at the level of 130 calories per kilogram of body weight per day. A standard water-soluble vitamin preparation was continued throughout the period of observation. Corn oil was given for three experimental periods in the form of a proprietary infant food.*

Collection of Freces. These were collected as described in a previous publication (Morales et al., 1950a).

Experimental Periods. These were of four days' duration in all instances.

Fat Amalyses. These were carried out on the faeces using the procedure of Tidwell and Holt (Tidwell and Holt, 1936).

\section{Results}

Table 1 shows the fat balances obtained in six infants when tributyrin and triacetin were substituted for butter fat. The superior absorption of tributyrin and triacetin is clearly shown; there was virtually complete absorption of both fats. The absorption of these two triglycerides was also superior to that of corn oil which had previously been shown by one of the present authors (Tidwell et al., 1935) to be better than butter fat.

Clinically, these infants did quite well on the short-chain fat feedings. There were, at no time, any untoward symptoms or signs that could be attributed to these feeds. Their weight gain on these feeds was in every instance better than on the butter-fat feed; it was inferior to that on the corn-oil feed because of the larger caloric value of the latter feeds. The comparative weight gains on these various forms of feed are shown in Table 2.

\footnotetext{
- Olac, kindly supplied by Mead Johnson \& Co.
} 
TABLE 1

FAT BALANCES IN SIX INFANTS

\begin{tabular}{|c|c|c|c|c|c|c|c|c|}
\hline \multirow{2}{*}{ Case } & & \multirow{2}{*}{$\begin{array}{c}\text { Age } \\
\text { (days) }\end{array}$} & \multirow{2}{*}{$\begin{array}{c}\text { Initial Weight } \\
\text { (g.) }\end{array}$} & \multirow{2}{*}{ Type of Fat } & \multirow{2}{*}{$\begin{array}{c}\text { Fat Intake } \\
\text { (g-/day) }\end{array}$} & \multirow{2}{*}{$\begin{array}{c}\text { Faecal Fat } \\
\text { (g./day) }\end{array}$} & \multicolumn{2}{|c|}{ Fat Retention } \\
\hline & & & & & & & (g/day) & $\begin{array}{l}\text { \% Intake } \\
\text { Retained }\end{array}$ \\
\hline Morrero |1 & $\cdots$ & 50 & 1,890 & $\begin{array}{l}\text { Tributyrin } \\
\text { Butter fat } \\
\text { Tributyrin }\end{array}$ & $\begin{array}{l}10 \cdot 8 \\
11 \cdot 5 \\
12 \cdot 0\end{array}$ & $\begin{array}{l}0 \cdot 08 \\
6 \cdot 44 \\
0 \cdot 22\end{array}$ & $\begin{array}{r}10 \cdot 72 \\
5 \cdot 06 \\
11 \cdot 78\end{array}$ & $\begin{array}{l}99.4 \\
44.0 \\
98.0\end{array}$ \\
\hline Camerero & $\cdots$ & 10 & 1,630 & $\begin{array}{l}\text { Tributyrin } \\
\text { Butter fat } \\
\text { Tributyrin }\end{array}$ & $\begin{array}{r}8 \cdot 0 \\
11 \cdot 5 \\
11 \cdot 5\end{array}$ & $\begin{array}{l}0 \cdot 15 \\
6 \cdot 69 \\
0 \cdot 30\end{array}$ & $\begin{array}{r}7 \cdot 85 \\
4 \cdot 81 \\
11 \cdot 20\end{array}$ & $\begin{array}{l}98 \cdot 2 \\
41 \cdot 9 \\
97 \cdot 3\end{array}$ \\
\hline Ford A .. & $\cdots$ & 5 & 1,350 & $\begin{array}{l}\text { Tributyrin } \\
\text { Butter fat } \\
\text { Tributyrin } \\
\text { Corn oil } \\
\text { Triacetin } \\
\text { Butter fat }\end{array}$ & $\begin{array}{c}6 \cdot 6 \\
7 \cdot 2 \\
7 \cdot 9 \\
8 \cdot 98 \\
10 \cdot 2 \\
10 \cdot 1\end{array}$ & $\begin{array}{l}0 \cdot 41 \\
4 \cdot 03 \\
0 \cdot 74 \\
2 \cdot 0 \\
0 \cdot 14 \\
1 \cdot 81\end{array}$ & $\begin{array}{r}6 \cdot 19 \\
3 \cdot 17 \\
7 \cdot 16 \\
6 \cdot 98 \\
10 \cdot 06 \\
8 \cdot 29\end{array}$ & $\begin{array}{l}93 \cdot 6 \\
44 \cdot 0 \\
90 \cdot 8 \\
77 \cdot 6 \\
98 \cdot 5 \\
82 \cdot 0\end{array}$ \\
\hline Ford $B$.. & $\cdots$ & 5 & 1,400 & $\begin{array}{l}\text { Tributyrin } \\
\text { Butter fat } \\
\text { Tributyrin } \\
\text { Corn oil } \\
\text { Triacetin } \\
\text { Butter fat }\end{array}$ & $\begin{array}{r}8 \cdot 0 \\
8 \cdot 4 \\
8 \cdot 7 \\
10 \cdot 0 \\
10 \cdot 2 \\
10 \cdot 9\end{array}$ & $\begin{array}{l}0 \cdot 10 \\
3 \cdot 82 \\
0 \cdot 10 \\
1 \cdot 27 \\
0 \cdot 15 \\
2 \cdot 25\end{array}$ & $\begin{array}{r}7 \cdot 90 \\
4 \cdot 58 \\
8 \cdot 60 \\
8 \cdot 73 \\
10 \cdot 05 \\
7 \cdot 65\end{array}$ & $\begin{array}{l}98 \cdot 8 \\
54 \cdot 5 \\
98 \cdot 9 \\
87 \cdot 3 \\
98 \cdot 3 \\
70 \cdot 3\end{array}$ \\
\hline Feliciano & $\cdots$ & 15 & 2,100 & $\begin{array}{l}\text { Triacetin } \\
\text { Butter fat } \\
\text { Triacetin } \\
\text { Corn oil }\end{array}$ & $\begin{array}{r}9 \cdot 9 \\
10 \cdot 6 \\
10 \cdot 6 \\
11 \cdot 8\end{array}$ & $\begin{array}{l}0 \cdot 09 \\
4 \cdot 35 \\
0 \cdot 10 \\
0 \cdot 8\end{array}$ & $\begin{array}{r}9 \cdot 81 \\
6 \cdot 25 \\
10 \cdot 50 \\
11 \cdot 0\end{array}$ & $\begin{array}{l}99 \cdot 3 \\
58 \cdot 8 \\
99 \cdot 0 \\
93 \cdot 2\end{array}$ \\
\hline Robbins & $\cdots$ & & & $\begin{array}{l}\text { Butter fat } \\
\text { Triacetin } \\
\text { Butter fat }\end{array}$ & $\begin{array}{r}8 \cdot 7 \\
9 \cdot 4 \\
10 \cdot 1\end{array}$ & $\begin{array}{l}2 \cdot 86 \\
0 \cdot 18 \\
4 \cdot 34\end{array}$ & $\begin{array}{l}5 \cdot 84 \\
9 \cdot 22 \\
6 \cdot 76\end{array}$ & $\begin{array}{l}67 \cdot 2 \\
98 \cdot 2 \\
66 \cdot 8\end{array}$ \\
\hline
\end{tabular}

TABLE 2

WEIGHT CHANGES

\begin{tabular}{|c|c|c|c|c|}
\hline Case & & Fat & & $\begin{array}{c}\text { Weight Change } \\
\text { (g-/day) }\end{array}$ \\
\hline Morrero & $\cdots$ & $\begin{array}{l}\text { Tributyrin } \\
\text { Butter fat } \\
\text { Tributyrin }\end{array}$ & & $\begin{array}{l}+28 \cdot 6 \\
+18 \cdot 3 \\
+20 \cdot 0\end{array}$ \\
\hline Camerero & . & $\begin{array}{l}\text { Tributyrin } \\
\text { Butter fat } \\
\text { Tributyrin }\end{array}$ & & $\begin{array}{l}-42 \cdot 5 \\
-3 \cdot 3 \\
-55 \cdot 0\end{array}$ \\
\hline Ford A .. & $\cdots$ & $\begin{array}{l}\text { Tributyrin } \\
\text { Butter fat } \\
\text { Tributyrin } \\
\text { Corn oil } \\
\text { Triacetin } \\
\text { Butter fat }\end{array}$ & & $\begin{array}{l}+25 \cdot 0 \\
-17 \cdot 5 \\
-20 \cdot 0 \\
-32 \cdot 8 \\
-15 \cdot 0 \\
-6 \cdot 7\end{array}$ \\
\hline Ford $B$.. & . & $\begin{array}{l}\text { Tributyrin } \\
\text { Butter fat } \\
\text { Tributyrin } \\
\text { Corn oil } \\
\text { Triacetin } \\
\text { Butter fat }\end{array}$ & & $\begin{array}{l}-24 \cdot 3 \\
-3 \cdot 3 \\
-23 \cdot 4 \\
-38 \cdot 4 \\
-20 \\
-16\end{array}$ \\
\hline Feliciano & . & $\begin{array}{l}\text { Triacetin } \\
\text { Butter fat } \\
\text { Triacetin } \\
\text { Corn oil }\end{array}$ & & $\begin{array}{l}-23 \cdot 4 \\
-1 \cdot 7 \\
\div 34 \cdot 2 \\
-31 \cdot 6\end{array}$ \\
\hline Robbins & . & $\begin{array}{l}\text { Butter fat } \\
\text { Triacetin } \\
\text { Butter fat }\end{array}$ & & $\begin{array}{l}-18 \cdot 4 \\
-35 \cdot 7 \\
-28\end{array}$ \\
\hline \multicolumn{3}{|c|}{$\begin{array}{l}\text { Average of } 8 \text { periods on tributyrin } \\
\text { Average of } 4 \text { periods on triacetin } \\
\text { Average of } 3 \text { periods on corn oil } \\
\text { Average of } 9 \text { periods on butter fat }\end{array}$} & $\begin{array}{l}\cdots \\
\cdots \\
\cdots\end{array}$ & $\begin{array}{l}-29 \cdot 9 \\
-28 \cdot 3 \\
-34 \cdot 3 \\
-11 \cdot 5\end{array}$ \\
\hline
\end{tabular}

\section{Comment}

The above studies indicate that the defect of fat absorption in premature infants can be overcome by the use of short-chain fats such as triacetin and tributyrin. This finding is a matter of scientific interest, suggesting as it does that the portal mechanism of fat absorption is unimpaired and that the lacteal mechanism alone is affected. As a matter of practical therapy, however, the substitution of the short-chain fats for butter appears to have little or no advantage from the point of view of supplying calories, since shortening the fatty acid chain involves a corresponding loss in energy yield. Tributyrin yields only 6.7 calories per gram and triacetin offers no appreciable caloric advantage over protein or carbohydrate.

\section{Summary}

Premature infants will absorb short-chain fats without difficulty, presumably because the portal absorption mechanism is unimpaired. The superior absorption of these fats is counterbalanced by their inferior caloric yield.

\section{REFERENCES}

Frazer, A. C. (1946). Physiol. Rev., 26, 103.

Gordon, H. H. and McNamara, H. (1941). Amer. J. Dis. Child., 62, 328.

Morales, S., Chung, A. W., Lewis, J. M., Messina, A. and Holt, L. E., Jr. (1950a). Pediatrics, 6, 86.

$\overline{\text { Muhi }} \overline{\text { G. }}$ (1923). $\overline{\text { Acta paediat Uppsala }}$ (1950), 6, 644.

Rubner, M. and Langstein, L. (1915). Arch. Anat. Physiol., Lpz. (Physiol. Abt.), p. 39.

Snyderman, S. E., Morales, S., Chung, A. W., Lewis, J. M., Messina, A. and Holt, L. E., Jr. (1953). Pediatrics, 12, 158.

Tidwell, H. C. and Holt, L. E., Jr. (1936), J. biol. Chem., 112, 605. 